\title{
Looking for patterns in the phytoplankton community of the Mediterranean microtidal Venice Lagoon: evidence from ten years of observations
}

\author{
FABRIZIO BERNARDI AUBRY, FRANCESCO ACRI, FRANCO BIANCHI \\ and ALESSANDRA PUGNETTI \\ National Research Council (CNR), Institute of Marine Science (ISMAR), Castello 1364/A, I-30122, Venezia, Italy. \\ E-mail: fabrizio.bernardi@ismar.cnr.it
}

\begin{abstract}
SUMMARY: By analysing a ten-year series (1998-2007) of data on hydrochemical properties, phytoplankton abundance and species composition in the Venice Lagoon, we identified i) the average annual phytoplankton biomass cycle, mainly unimodal and fairly well tuned with the fluctuations in temperature and irradiance and (ii) the seasonal succession of the most important species, allowing us to construct a "phytoplankton calendar". Phytoplankton biomass was significantly lower in the second half of the ten-year period (2003-2007) than in the first half (1998-2002).
\end{abstract}

Keywords: phytoplankton community, multiannual variation, seasonal pattern, spatial variability, Venice Lagoon, Adriatic Sea.

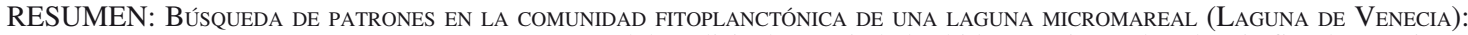
EVIDENCIAS EN DIEZ AÑOS DE OBSERVACIÓN. - A través del análisis de propiedades hidroquímicas, abundancia fitoplanctónica y composición de especies, en series de diez años (1998-2007), en la laguna de Venecia, se han identificado: i) Un promedio anual del ciclo de la biomasa prevalentemente de forma unimodal y bien sintonizada con las fluctuaciones de temperatura e irradiación; ii) Una composición cualitativa del fitoplancton que nos ha permitido realizar un "calendario del fitoplancton"; iii) Un decrecimiento en los últimos 5 años (2003-2007), de la biomasa con respecto a los 5 primeros años (1998-2003).

Palabras clave: comunidad fitoplanctónica, variación multi-anual, patrón estacional, variabilidad espacial, laguna de Venecia, Adriatico.

\section{INTRODUCTION}

The study of the seasonal phytoplankton cycle is crucial for identifying temporal shifts related to climate variability or local impacts. The annual phytoplankton cycle differs across aquatic ecosystems and patterns of biomass variation often do not closely follow annual climate change (Winder and Cloern 2010). A variety of phytoplankton seasonal cycles are found in transitional and coastal ecosystems (Cloern and Jassby 2008), so recognition of recurring spatial and temporal patterns often represents a challenging task. Some traits of these ecosystems, such as shallowness, close benthic-pelagic coupling and connectivity to both land and sea, affect phytoplankton composition and distribution on both spatial and temporal scales (Cloern and Jassby 2010). The complexity of these areas and the exposure of phytoplankton to the climate cycle and a range of local drivers explain the heterogeneity of annual fluctuations (Winder and Cloern 2010).

On the mesoscale at least, a small number of climatic factors may be recognized as the main drivers of the seasonal phytoplankton cycle in aquatic ecosystems such as open seas and large lakes. Here, the seasonal succession of phytoplankton species and biomass variation shows some regularities, which can be 
more easily assessed than in transitional environments and even predicted in some cases. The phytoplankton community of transitional waters appears to be characterized by a pronounced degree of unpredictability, making it harder to define "baselines" against which to evaluate local vs large-scale changes as well as multiannual trends (Edwards et al. 2010). The high noiseto-signal ratio and the multiple-process and multiplescale regulation of phytoplankton complicates the task of detecting climate-driven trends. In this respect, the availability of multiannual ecological series, gathered with appropriate methods and on suitable scales of investigation, is a unique and invaluable tool.

The intrinsically high variability of phytoplankton communities in transitional environments should be taken into account not only because of phytoplankton ecological importance, the definition of community patterns being a critical question in ecology, but also because of the implications for environmental management. Indeed, phytoplankton is the only planktonic element included as a water quality indicator in the European Water Framework Directive (WFD 2000/60/ EC). Some phytoplankton-related variables (composition, abundance, biomass, frequency and intensity of blooms) are essential for the definition and the classification of water quality.

The Venice Lagoon (Fig. 1) is the largest Italian lagoon and is included in the Italian Long Term Ecosystem Research network (LTER-Italy). Data on phytoplankton composition in the Lagoon date back to the 1970s (Voltolina 1975, Socal et al. 1985, Socal et al. 1987, Tolomio et al. 1999, Bernardi Aubry and Acri 2004, Acri et al. 2004, Socal et al. 2006). The phytoplankton assemblage is dominated by diatoms: neritic species, adapted to survival over large salinity ranges, and tychopelagic species, suspended due to hydrodynamics (Socal et al. 1985; Tolomio and Bullo 2001). Neritic species (dinoflagellates or coccolithophorids) are often introduced by saline waters at flood tide, whereas at ebb tide we find oligohaline species (chlorophyceans, euglenophyceans) in the inner areas of the Lagoon (Socal et al. 1987). Most studies have considered restricted areas of the Lagoon and only one-year cycles; repeated long-term observations only started in 1998. In transitional water ecosystems, primary producers are usually highly diversified, involving several functional levels and types (phytoplankton, benthic microalgae, aquatic angiosperms and macroalgae). At present, considering the whole basin, the bed of the Venice Lagoon appears to be dominated by seagrasses, particularly Cymodocea nodosa (Sfriso et al. 2005, Sfriso and Facca 2007). However, in some areas, phytoplankton is the main primary producer (Acri et al. 2004, Sfriso et al. 2005). The focus of the present paper is the analysis of a ten-year series (1998-2007) of monthly data on the phytoplankton community and the main related abiotic factors. Our main aim is to identify mesoscale phytoplankton patterns and trends in the Lagoon of Venice by considering: i) spatial variability, ii) seasonal dynamics, and iii) multiannual series.

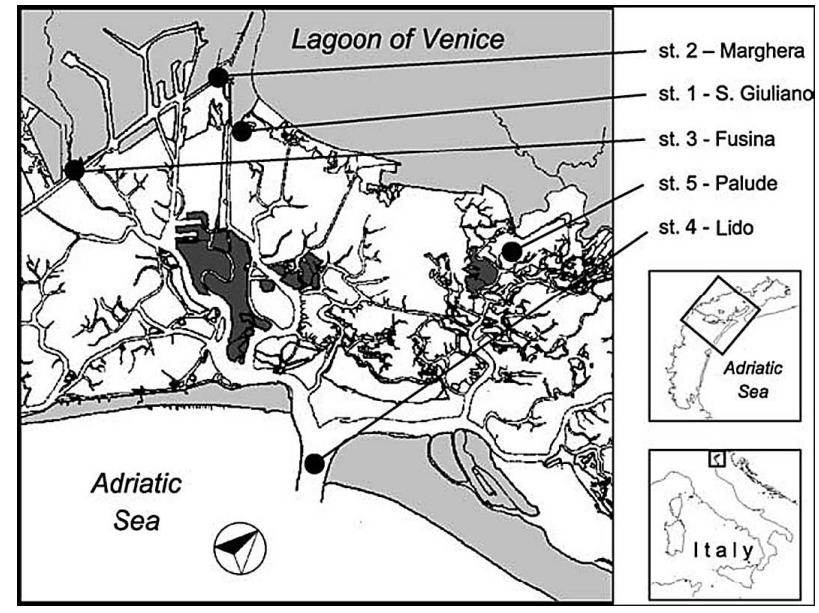

FIG. 1. - Venice Lagoon and five sampling stations.

\section{MATERIALS AND METHODS}

\section{Study area and sampling strategy}

The Lagoon of Venice (Fig. 1) is a large Mediterranean lagoon $\left(550 \mathrm{~km}^{2}\right)$ in the northern Adriatic Sea. It is delimited by densely inhabited shores and industrial areas and is affected by high tourist flows, fisheries and aquaculture. It has an average depth of $1 \mathrm{~m}$ and is morphologically characterized by the presence of large shallow areas and a network of deeper $(5-10 \mathrm{~m})$ channels. It is separated from the Adriatic Sea by sandbars, which are interrupted by three inlets. Water turnover in the Lagoon is maintained by the tidal cycle through the inlets. Water circulation results from the interactions of tide, wind and topography (Solidoro et al. 2004, Gačić et al. 2005); water renewal ranges from a few days, close to the inlets, to one month in the inner areas (Solidoro et al. 2004, Cucco and Umgiesser 2006). The tidal amplitude is $100 \mathrm{~cm}$, with maxima of $150 \mathrm{~cm}$. The Lagoon can be classified as polyhaline. Twelve main tributaries discharge an annual average of about $35 \mathrm{~m}^{3} \mathrm{~s}^{-1}$ of freshwater into the Lagoon with seasonal peaks in spring and autumn (ARPAV 2009) and the monthly maximum in November (55.5 $\mathrm{m}^{3} \mathrm{~s}^{-1}$; Zuliani et al. 2005). Nitrogen and phosphorus loading is of the order of $4000 \mathrm{t}^{\text {year }}{ }^{-1}$ and $230 \mathrm{t}$ year $^{-1}$, respectively (Collavini et al. 2005). The Venice Lagoon presents marked habitat heterogeneity and habitat classification is still a matter of debate (Tagliapietra et al. 2009).

For ten years monthly samplings were taken at five stations (120 monthly surveys in total from 1998 to 2007). These were selected so as to be representative of the natural and anthropogenic environmental variability of the northern and central basins of the Lagoon (Fig. 1), which are characterized by a complex interplay between freshwater and marine inputs (Cucco and Umgiesser 2006; Solidoro et al. 2004) and by anthropogenic impacts (Bianchi et al. 2003). The stations are located in areas where the presence of macrophytes is negligible 
and most primary production is by phytoplankton (Acri et al. 2004, Sfriso et al. 2005). Station 1, S. Giuliano (depth $2 \mathrm{~m}$ ), is an area of intense maritime traffic (Bianchi et al. 1996, Socal et al. 1999); it collects urban waste from the town of Mestre, and receives freshwater inputs from a small channel. Station 2, Marghera (depth $9.5 \mathrm{~m}$ ), receives both seawater inputs, during the tide, from the Lido inlet, and fresh waters from a channel; it is also affected by pollutants of industrial origin (Perin 1975, Guerzoni et al. 2007). Station 3, Fusina (depth 3.5 $\mathrm{m})$, is affected by both freshwater and marine inputs and by heat emissions from the Porto Marghera power station (Alberighi et al. 1992, Socal et al. 1999). Station 4, Lido (depth $7 \mathrm{~m}$ ), is located in the northernmost inlet of the Lagoon under the direct influence of Adriatic coastal waters. Station 5, Palude (depth $2.7 \mathrm{~m}$ ), is in an inland marshy area, a typical lagoon environment, influenced both by fresh waters entering from a channel and, to a lesser extent, by sea water carried by the tide (Bianchi et al. 1999). At every station measurements and samplings were performed at neap tide. Only the surface water layer was sampled, vertical phytoplankton distribution and the abiotic structure of the water column lying outside the scope of this study. The water column was assumed to be well mixed, irregular measurements carried out along the water column indicating negligible salinity and temperature differences between surface and bottom.

\section{Measured variables}

During each survey we measured transparency with Secchi disks; temperature, with a bucket thermometer; salinity, with a Guildline Autosal 8400B laboratory salinometer; dissolved oxygen, in accordance with Winkler's method (Strickland and Parsons 1972); dissolved nutrients including nitrogen as ammonium $\left(\mathrm{N}-\mathrm{NH}_{4}\right)$, nitrites $\left(\mathrm{N}-\mathrm{NO}_{2}\right)$ and nitrates $\left(\mathrm{N}-\mathrm{NO}_{3}\right)$, phosphorus as orthophosphates $\left(\mathrm{P}-\mathrm{PO}_{4}\right)$ and silica as orthosilicates $\left(\mathrm{Si}_{-} \mathrm{SiO}_{4}\right)$, all measured after filtering through Whatman GF/F glass fibre filters and analysed with a Systea-Alliance auto-analyser (Strickland and Parsons1972; Hansen and Koroleff 1999); chlorophyll $a$ (chl $a$; Holm-Hansen et al. 1965), after filtering with Whatman GF/F filters and measurement of the acetone extract by Perkin Elmer LS5B spectrofluorometer before and after acidification; and phytoplankton, fixed in neutralized formalin with hexamethylentetramine, recognized and counted with an invertoscope (Uthermöhl 1958, Zingone et al. 2010). Species composition was mainly defined in accordance with Tomas (1997). Determinations for specific algal groups were also carried out with reference to Peragallo (1897-1908), Hustedt (1930-1966), Sournia (1986) and Hendey (1964) for diatoms; Schiller (1931-1937), Rampi and Bernhard (1980) and Sournia (1986) for dinoflagellates; Throndsen (1993) for dictyophyceans; Rampi and Bernhard (1981) and Heimdal (1993) for coccolithophorids; Throndsen (1993) for euglenohyceans; and Pascher (1915) for chlorophyceans.
Phytoplankton analysis was confined only to those forms that were detectable by light microscopy, so the phytoplankton fraction $<3 \mu \mathrm{m}$ (picophytoplankton) was not considered. The 'nanoflagellates' group included all the undetermined organisms whose sizes varied between 3 and $4 \mu \mathrm{m}$, consisting of cryptophyceans, chrysophyceans, prymnesiophyceans (except coccolithophorids), prasinophyceans, and chlorophyceans. Incident irradiance was obtained from the ISMAR-CNR meteorological archive.

\section{Statistical analyses}

Statistical analyses (ANOVA, Kruskall-Wallis, Mann-Whitney test and correlation analysis) were performed using Statistica by Statsoft Kernel release 5.5 , after log-transformation of biological data (Cassie, 1962) to account for non-normal data distributions (Sokal and Rohlf 1981). We used a matrix composed of 216 taxa and 600 samples for describing spatial differences among stations and temporal (both seasonal and interannual) dynamics. We used the Shannon index (Shannon and Weaver 1949) to estimate phytoplankton biodiversity. This index increases with the number of species (with the above-mentioned qualifications regarding microscopic determination) and the evenness of their distribution. In order to analyse the spatial and temporal pattern of community structure, the original phytoplankton abundance data were used to produce data matrices and perform multivariate analyses using PRIMER 5.2.2 software. The multi-dimensional scaling (MDS) ordination method was used on the speciessamples matrix, after a double square root transformation of abundance data (Clarke and Warwick 1994; Carr 1996).

Redundancy analysis (RDA; CANOCO 4.53 software) was used to identify relations between phytoplankton taxa and environmental variables. RDA is an extension of multiple regression and it is used when there is more than one response variable (here, the taxa). RDA was used to analyse the environmental and biological data together, with taxa ordination related to a combination of environmental variables (Legendre and Legendre 1998). Within this analysis, the relative proximity of samples, or averaged groups of samples, reflects relative similarity in species composition. The environmental variables are represented by vectors: their length indicates the magnitude of the correlation between the environmental variables and the phytoplankton species. The most characteristic species or species assemblages of each group of samples (stations, seasons, years or samplings) were considered to be "indicator species". These were identified using INDVal 2.0 software (http://biodiversite.wallonie.be/ outils/indval/), considering those species or assemblages that were found mostly in a single group or at the majority of the sites belonging to that group (Dufrêne and Legendre 1997). For each species $i$ in each group of sites $j$, we derived IndVal $\mathrm{Va}_{i j}$ from $\mathrm{A}_{i j}$ (the mean 
abundance of species $i$ in the sites of group $j$ ) and $\mathrm{B}_{i j}$ (the relative frequency of occurrence of species $i$ in the sites of group $j$ ) as follows:

$$
\begin{gathered}
\mathrm{A}_{i j}=\mathrm{N}_{\text {individuals }} / \mathrm{N}_{i j} \text { individuals }_{i} ; \\
\mathrm{B}_{i j}=\mathrm{Nsites}_{i j} / \mathrm{Nsites}_{j} ; \\
\operatorname{IndVal}_{i j}=\mathrm{A}_{i j} \mathrm{~B}_{i j} \times 100
\end{gathered}
$$

where IndVal is the Indicator Value of species $i$ in site cluster $j$. The statistical significance of the species indicator values was assessed using a randomization procedure. The species that best characterize each group are those with the highest IndVal.

\section{Spatial variability}

In order to provide a clearer picture of the study area, the sampling sites were aggregated into Zones of Similar Influence (ZSIs; Boyer et al. 1997) based on cluster analysis of the main hydrochemical parameters: temperature, salinity, DIN as a sum of N-NH and $\mathrm{N}-\mathrm{NO}_{3}, \mathrm{P}_{-} \mathrm{PO}_{4}, \mathrm{Si}_{-} \mathrm{SiO}_{4}$ and chl $a$. MDS analysis was then used to define spatial differences dependent on the qualitative composition of phytoplankton populations in the different sampling sites. The various ZSIs were compared using the Kruskall-Wallis nonparametric test, the key result of which is a $p$ value that indicates whether groups of samples have the same median.

\section{Temporal variability}

RDA analysis was used to define temporal differences between phytoplankton populations in the different years or groups of years and to relate them to the main environmental parameters; values for phytoplankton species abundance and environmental variables were averaged for the stations sampled. We defined the seasonal categories as follows: winter (January-March), spring (April-June), summer (July-September) and autumn (October-December). For the interannual trends, we applied seasonal Kendall- $\tau$ non-parametric analysis to the whole data set using WQ2 software (release 2.0 by EQMetric, LLC and Virginia Tech Intellectual
Properties Inc.). This analysis determines the direction of trends $(+$ or -$)$, goodness of fit $(\tau)$ and the statistical significance of fit (Boyer et al. 1999, Hirsch et al. 1991). The rate of change of each variable is quantified by the seasonal Kendall slope: the median (over all pairs of years for each month) of the change-per-year of each parameter (Theil 1950, Sen 1968). To compare different groups of samples (years), we performed the Mann-Whitney non-parametric test, the key result of which is a $p$ value that indicates whether two groups of samples have the same median.

\section{Bloom definition}

"Blooms" were defined as cases dominated by a single species (abundance $>50 \%$ ) and chl $a>10 \mu \mathrm{g} \mathrm{l}^{-1}$ (see also Sin et al. 1999, Gameiro et al. 2007), the latter corresponding to mean abundance of $23 \times 10^{6}{\text { cells } 1^{-1}}^{-1}$ (standard deviation $17 \times 10^{6}$ cells $1^{-1}$ ). The bloom frequency at each station, in each season and throughout the period was simply calculated as the ratio between the number of bloom samples and the total.

\section{RESULTS}

From a total of 600 phytoplankton samples we identified 216 distinct taxa belonging to 10 divisions: diatoms (141), dinoflagellates (40), chlorophyceans (21), coccolithophorids (10), chrysophyceans and euglenohyceans (4). Identifiable dictyophyceans, chlorophyceans, chryptophyceans, and prasinophyceans accounted for less than 4 taxa each; bearing in mind that most of the individuals from these divisions were unidentifiable at species level, in this study they were included in the group 'nanoflagellates'.

Diatoms and nanoflagellates dominated the community, accounting for $61 \%$ and $32 \%$ of abundance, respectively, while cryptophyceans, dinoflagellates, chlorophyceans and euglenophyceans accounted for $1 \%$ to $3 \%$. All other taxa (coccolithophorids, dictiochophyceans, prymnesiophyceans) accounted for less than $1 \%$, although they were abundant in some individual samples. A small number of species dominated the community: the "top ten" accounted for $88 \%$ of

TABLE 1. - List of "top ten" phytoplankton taxa recorded throughout study period at all stations, with average (whole dataset) abundance,

\begin{tabular}{|c|c|c|c|c|c|c|}
\hline & Species & Author & $\begin{array}{l}\text { Abundance } \\
\left(\text { cells } 1^{-1}\right)\end{array}$ & $\begin{array}{l}\text { Standard } \\
\text { deviation } \\
\left(\text { cells } 1^{-1}\right)\end{array}$ & $\begin{array}{c}\text { Abundance } \\
\text { contribution } \\
(\%)\end{array}$ & $\begin{array}{r}\text { Cumulative } \\
\text { abundance } \\
\text { contribution (\%) }\end{array}$ \\
\hline Diatoms & Thalassiosira sp. & & 897756 & 4412277 & 29.0 & 29.0 \\
\hline Diatoms & Nitzschia frustulum & Grunow 1862 & 505596 & 3690909 & 16.4 & 45.4 \\
\hline Diatoms & Skeletonema marinoi & Sarno and Zingone 2005 & 468276 & 1993843 & 15.1 & 60.6 \\
\hline Diatoms & Cyclotella $\mathrm{sp}$. & & 259555 & 1795480 & 8.4 & 68.9 \\
\hline Diatoms & Cylindrotheca closterium & (Ehrenberg) Lewin and Reimann 1964 & 189563 & 1219608 & 6.1 & 75.1 \\
\hline Cryptophyceans & Und. Cryptophyceae & & 143521 & 388876 & 4.6 & 79.7 \\
\hline Diatoms & Chaetoceros sp. & & 92319 & 504805 & 3.0 & 82.7 \\
\hline Diatoms & Navicula sp. & & 68147 & 160299 & 2.2 & 84.9 \\
\hline Diatoms & Chaetoceros compressus & Lauder 1864 & 54183 & 570080 & 1.8 & 86.7 \\
\hline Diatoms & Navicula cryptocephala & (Kützing) Kützing 1844 & 35585 & 107255 & 1.2 & 87.8 \\
\hline
\end{tabular}
standard deviations and relative and cumulative percentage of each taxon. Cumulative abundance is the sum of relative contribution of abundance of a single species. 
TABLE 2. - Averages (AVG) and standard deviations (SD) for whole study period of main abiotic parameters Shannon index and phytoplankton biomass and abundance at each station.

\begin{tabular}{|c|c|c|c|c|c|c|c|c|c|c|}
\hline & \multicolumn{2}{|c|}{$\begin{array}{c}\text { Station } 1 \\
\text { San Giuliano }\end{array}$} & \multicolumn{2}{|c|}{$\begin{array}{c}\text { Station } 2 \\
\text { Marghera }\end{array}$} & \multicolumn{2}{|c|}{$\begin{array}{l}\text { Station } 3 \\
\text { Fusina }\end{array}$} & \multicolumn{2}{|c|}{$\begin{array}{c}\text { Station } 4 \\
\text { Lido }\end{array}$} & \multicolumn{2}{|c|}{$\begin{array}{c}\text { Station } 5 \\
\text { Palude }\end{array}$} \\
\hline & $\mathrm{AVG}$ & $\mathrm{SD}$ & AVG & $\mathrm{SD}$ & AVG & SD & AVG & $\mathrm{SD}$ & AVG & $\mathrm{SD}$ \\
\hline Temperature $\left({ }^{\circ} \mathrm{C}\right)$ & 16.2 & 7.9 & 18.0 & 6.8 & 22.4 & 5.4 & 16.4 & 7.0 & 15.5 & 8.1 \\
\hline Salinity & 23.77 & 5.51 & 30.03 & 2.26 & 29.60 & 2.87 & 33.76 & 2.29 & 26.91 & 4.55 \\
\hline $\mathrm{N}^{-\mathrm{NH}_{4}}(\mu \mathrm{M})$ & 18.01 & 12.80 & 16.74 & 12.55 & 13.70 & 7.80 & 4.08 & 3.79 & 9.32 & 6.48 \\
\hline $\mathrm{N}-\mathrm{NO}_{2}(\mu \mathrm{M})$ & 2.12 & 1.35 & 1.80 & 0.97 & 1.48 & 0.68 & 0.70 & 0.66 & 1.43 & 0.82 \\
\hline $\mathrm{N}-\mathrm{NO}_{3}(\mu \mathrm{M})$ & 41.93 & 33.51 & 32.53 & 22.00 & 37.66 & 21.16 & 16.21 & 12.73 & 38.55 & 34.60 \\
\hline DIN $(\mu \mathrm{M})$ & 62.06 & 41.35 & 51.07 & 31.60 & 52.84 & 23.92 & 20.99 & 14.15 & 49.30 & 37.64 \\
\hline $\mathrm{SI}_{-S I O}(\mu \mathrm{M})$ & 51.84 & 34.52 & 30.09 & 31.05 & 32.75 & 24.47 & 21.81 & 32.62 & 48.85 & 44.25 \\
\hline $\mathrm{P}_{-} \mathrm{PO}_{4}(\mu \mathrm{M})$ & 1.52 & 1.00 & 1.09 & 0.96 & 1.02 & 0.48 & 0.13 & 0.12 & 0.36 & 0.47 \\
\hline Shannon (bit) & 0.72 & 0.27 & 0.61 & 0.28 & 0.76 & 0.26 & 0.79 & 0.22 & 0.82 & 0.22 \\
\hline Chlorophyll $a\left(\mu \mathrm{g} \mathrm{l}^{-1}\right)$ & 12.66 & 19.68 & 9.47 & 14.48 & 3.38 & 4.56 & 2.28 & 4.59 & 4.37 & 9.34 \\
\hline Total phytoplankton Abundance (cells $1^{-1}$ ) & 8444779 & 14821876 & 74751611 & 11818155 & 2671208 & 4060586 & 1618115 & 2280961 & 3060024 & 5178748 \\
\hline Diatoms (cells $1^{-1}$ ) & 5855717 & 12070027 & 4513282 & 9494444 & 1384491 & 2385960 & 640005 & 1113899 & 1748657 & 4012019 \\
\hline Dinoflagellates (cells $1^{-1}$ ) & 97637 & 336745 & 146527 & 738125 & 37127 & 95888 & 50754 & 117055 & 26982 & 55700 \\
\hline Coccolitophorids (cells $1^{-1}$ ) & 2576 & 8037 & 3104 & 13676 & 5618 & 33161 & 23424 & 68486 & 4019 & 11990 \\
\hline Cryptophyceae (cells $1^{-1}$ ) & 122576 & 281929 & 226174 & 415833 & 55694 & 95190 & 181320 & 611632 & 149205 & 371411 \\
\hline Chrysophyceae (cells $1^{-1}$ ) & 326 & 3510 & 4408 & 46405 & 0 & 0 & 339 & 1759 & 0 & 0 \\
\hline Chlorophyceae (cells $1^{-1}$ ) & 42113 & 209973 & 25465 & 131144 & 113243 & 649804 & 2239 & 16083 & 16372 & 59112 \\
\hline Euglenophyceae (cells $1^{-1}$ ) & 122769 & 971318 & 38793 & 93201 & 3759 & 16170 & 3412 & 20183 & 5147 & 30968 \\
\hline Prasinophyceae (cells $\left.1^{-1}\right)$ ) & 3819 & 23470 & 10880 & 49614 & 1039 & 6781 & 2170 & 8972 & 3530 & 20956 \\
\hline Prymnesiophyceae (cells $1^{-1}$ ) & 141 & 1517 & 831 & 9023 & 0 & 0 & 465 & 5005 & 0 & 0 \\
\hline Dictyochophyceae (cells $1^{-1}$ ) & 0 & 0 & 91 & 992 & 431 & 4659 & 79 & 483 & 0 & 0 \\
\hline Nanoflagellates (cells $1^{-1}$ ) & 2141949 & 4244353 & 2505606 & 5586118 & 1069809 & 2188504 & 713910 & 933176 & 1105607 & 1564852 \\
\hline Cyanophyceae (cells $1^{-1}$ ) & 55157 & 581651 & 0 & 0 & 0 & 0 & 0 & 0 & 506 & 3917 \\
\hline
\end{tabular}

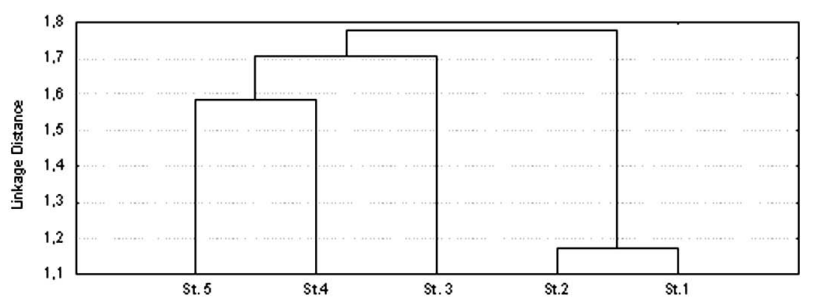

FIG. 2. - Cladogram identifying three Zones of Similar Influence (ZSI) based on environmental parameters (temperature, salinity, nutrients) and chl $a$.

cumulative abundance (Table 1), while fewer than 60 species accounted for $99 \%$.

\section{Habitat heterogeneity and phytoplankton spatial variability}

Phytoplankton was sampled across a wide range of environmental conditions (Table 2), as demonstrated by the variability of temperature $\left(0.6^{\circ} \mathrm{C}\right.$ to $\left.29.9^{\circ} \mathrm{C}\right)$, salinity (8.6-37.1) and nutrients (DIN 1.2-243.9 $\mu \mathrm{M}$;

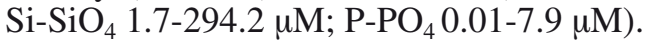

TABLE 3. - Kruskall-Wallis test of abiotic parameters and chl $a$, highlighting differences among three ZSIs (N, number of samples).

\begin{tabular}{lcrcr}
\hline & St. 1-2 & \multicolumn{1}{c}{ St. 3 } & St. 4-5 & p level \\
\hline Salinity & 28.35 & 30.12 & 31.36 & 0.01 \\
Temperature $\left({ }^{\circ} \mathrm{C}\right)$ & 18.35 & 24.15 & 16.55 & 0.01 \\
$\mathrm{DIN}(\mu \mathrm{M})$ & 47.73 & 49.43 & 27.37 & 0.01 \\
$\mathrm{Si}^{-S_{i}}(\mu \mathrm{M})$ & 31.8 & 23.63 & 21.05 & 0.01 \\
$\mathrm{P}_{4} \mathrm{PO}_{4}(\mu \mathrm{M})$ & 1.1 & 0.91 & 0.14 & 0.01 \\
$\left.\mathrm{Chl} a(\mu \mathrm{g})^{-1}\right)$ & 3.81 & 1.75 & 1.49 & 0.01 \\
$\mathrm{~N}$ & 240 & 120 & 240 & \\
\hline
\end{tabular}

Based on the main abiotic parameters (temperature, salinity, DIN as a sum of $\mathrm{N}-\mathrm{NH}_{4}, \mathrm{~N}-\mathrm{NO}_{2}$ and $\mathrm{N}-\mathrm{NO}_{3}$, $\mathrm{P}-\mathrm{PO}_{4}$, and $\mathrm{Si}-\mathrm{SiO}_{4}$ ) and chl $a$, the five sampling sites were aggregated into three ZSIs (Fig. 2).

The highest phytoplankton biomass and the lowest salinity were found at stations 1 and 2, station 3 had the highest temperature, and stations 4 and 5 relatively low nutrient concentrations $\left(\mathrm{Si}-\mathrm{SiO}_{4}, \mathrm{P}_{-} \mathrm{PO}_{4}\right.$ and $\mathrm{DIN}$, Table 3). In contrast, phytoplankton species composition at the five stations did not exhibit significant differences (Fig. 3). From the qualitative point of view, the most abundant species appear to be largely shared by all stations. Indeed, as indicated by IndVal analysis (Table 4), the differences between the stations are mainly due to: 1) marked dissimilarities in the abundance and frequency of a pool of taxa common to all stations (see "top ten", Table 1 and Table 4); and 2) the presence of specific taxa that are associated, albeit to different degrees, with individual stations (Table 4). Considering this last group, station 4 is mainly typified by a marine community, with species that are also found in the coastal waters of the northern Adriatic Sea (Bernardi Aubry et al. 2004) but are usually infrequent inside the Lagoon: the diatoms Proboscia alata, Pseudo-nitzschia delicatissima and Cerataulina pelagica, the dinoflagellates Gyrodinium sp. and the coccolithophorid Emiliania huxleyi. Even rarer and exclusive or nearly exclusive to this station are the dinoflagellates Neoceratium furca, the diatom Guinardia striata and the coccolitophorids Rhabdosphaera claviger, Syracosphaera pulcra and Calciosolenia murrayi. Station 2 is characterized by a few flagellate species (nanoflagellates and dinoflagellates such as Gymnodinium sp.), the diatom Skeletonema marinoi and some species, such as Eutreptia lanowii, that typically inhabit fresh waters but 
TABLE 4. - IndVal for "top ten" taxa (A) and other taxa (B): significant IndVal values are highlighted for each station.

\begin{tabular}{|c|c|c|c|c|c|c|}
\hline & Station 1 & Station 2 & Station 3 & Station 4 & Station 5 & p level \\
\hline \multicolumn{7}{|l|}{ A) } \\
\hline Thalassiosira spp. & 42.0 & 30.0 & 9.0 & 1.2 & 2.7 & 0.003 \\
\hline Cylindrotheca closterium & 33.3 & 3.6 & 5.8 & 2.1 & 7.3 & 0.007 \\
\hline Nitzschia frustulum & 17.7 & 0.5 & 1.3 & 0.06 & 12.5 & 0.011 \\
\hline Skeletonema marinoi & 5.6 & 46.1 & 6.6 & 0.8 & 0.2 & 0.000 \\
\hline Chaetoceros sp. & 2.6 & 11.1 & 1.1 & 4.3 & 2.9 & 0.046 \\
\hline Und. Cryptophyceae & 12.7 & 27.1 & 6.3 & 23.0 & 16.7 & 0.050 \\
\hline Cyclotella sp. & 7.0 & 7.6 & 3.3 & 1.8 & 1.0 & 0.7 \\
\hline Chaetoceros compressus & 0.02 & 3.1 & 0.2 & 4.1 & 0.04 & 0.127 \\
\hline Navicula sp. & 33.7 & 3.9 & 11.7 & 2.9 & 34.7 & 0.000 \\
\hline $\begin{array}{l}\text { Navicula cryptocephala } \\
\text { B) }\end{array}$ & 30.8 & 1.4 & 3.6 & 0.6 & 35.5 & 0.000 \\
\hline Und. Pennatae & 21.6 & 0.9 & 4.1 & 0.7 & 5.6 & 0.001 \\
\hline Gyrosigma fasciola & 17.3 & 0.01 & 0.09 & 0.02 & 1.6 & 0.000 \\
\hline Cocconeis sp. & 12.0 & 0.5 & 4.1 & 2.4 & 8.8 & 0.014 \\
\hline Nitzschia conscricta & 9.9 & 0.5 & 1.5 & 0.01 & 2.2 & 0.003 \\
\hline Gomphonema olivaceum & 6.7 & 0.3 & 1.1 & 0.003 & 0.005 & 0.001 \\
\hline Gomphonema parvulum & 5.9 & 0.02 & 1.5 & 0.04 & 0.1 & 0.003 \\
\hline Skeletonema marinoi & 5.6 & 46.1 & 6.6 & 0.9 & 0.2 & 0.000 \\
\hline Nanoflagellates & 28.1 & 32.7 & 13.9 & 9.6 & 14.5 & 0.002 \\
\hline Gymnodinium sp. & 9.4 & 20.8 & 3.6 & 12.5 & 4.4 & 0.002 \\
\hline Eutreptia lanowii & 12.8 & 14.4 & 0.1 & 0.06 & 0.05 & 0.000 \\
\hline Scenedesmus quadricauda & 0.9 & 0 & 8.2 & 0.002 & 0.03 & 0.000 \\
\hline Aulacoseira granulata & 0.03 & 0 & 6.8 & 0 & 0.2 & 0.000 \\
\hline Hermesinum adriaticum & 0 & 0 & 2.5 & 0 & 0 & 0.005 \\
\hline Emiliania huxleyi & 0.7 & 1.4 & 3.3 & 33.3 & 1.9 & 0.000 \\
\hline Cerataulina pelagica & 0.005 & 0.2 & 0.5 & 24.6 & 0.1 & 0.000 \\
\hline Pseudo-nitzschia delicatissima & 0.4 & 0.8 & 1.0 & 23.8 & 1.4 & 0.000 \\
\hline Proboscia alata & 0 & 0.05 & 0.02 & 14.2 & 0.07 & 0.000 \\
\hline Syracosphaera pulchra & 0 & 0 & 0.006 & 11.3 & 0.6 & 0.000 \\
\hline Und. Coccolitophorids & 0.4 & 0.7 & 0.3 & 9.7 & 0.1 & 0.000 \\
\hline Leptocylindrus danicus & 0.07 & 0.9 & 0.03 & 9.6 & 0.1 & 0.000 \\
\hline Asterionellopsis glacialis & 0.01 & 0.03 & 0.03 & 9.0 & 0.09 & 0.000 \\
\hline Prorocentrum micans & 0.008 & 2.0 & 0.05 & 7.9 & 0.03 & 0.000 \\
\hline Chaetoceros decipiens & 0 & 1.0 & 0.02 & 6.5 & 0.07 & 0.001 \\
\hline Gyrodinium sp. & 0.9 & 0.01 & 0.4 & 5.2 & 0.06 & 0.008 \\
\hline Guinardia striata & 0.09 & 0 & 0 & 5.1 & 0 & 0.001 \\
\hline Neoceratium furca & 0 & 0 & 0 & 4.1 & 0 & 0.000 \\
\hline Rhabdosphaera claviger & 0 & 0 & 0 & 3.2 & 0 & 0.005 \\
\hline Calciosolenia murrayi & 0 & 0 & 0.03 & 3.1 & 0 & 0.006 \\
\hline Amphora sp. & 21.8 & 2.7 & 8.1 & 2.4 & 37.0 & 0.000 \\
\hline Amphora exigua & 12.2 & 1.7 & 2.0 & 1.1 & 24.2 & 0.000 \\
\hline Amphora coffaeformis & 9.9 & 0.3 & 1.1 & 1.1 & 20.3 & 0.000 \\
\hline Cocconeis scutellum & 16.7 & 1.5 & 6.3 & 0.9 & 17.1 & 0.003 \\
\hline Psammodictyon panduriforme & 1.4 & 0.6 & 2.6 & 0.03 & 15.5 & 0.000 \\
\hline Halamphora veneta & 2.5 & 0.8 & 1.4 & 0.8 & 14.2 & 0.000 \\
\hline Amphora hyalina & 0.05 & 0 & 0 & 0 & 12.7 & 0.000 \\
\hline Licmophora gracilis & 0.01 & 0 & 1.5 & 0.03 & 7.4 & 0.000 \\
\hline Amphora lineolata & 0.01 & 0 & 0.04 & 0 & 7.1 & 0.000 \\
\hline Amphora laevissima & 0 & 0 & 0 & 0 & 5.7 & 0.000 \\
\hline
\end{tabular}

are also fairly widespread in coastal waters. Freshwater species (the diatom Aulacoseira granulata and the chlorophycean Scenedesmus quadricauda) characterize station 3. Benthic-resuspended diatoms (e.g. Halamphora exigua, Amphora coffaeformis, Cocconeis scutellum, Cocconeis sp., Cylindrotheca closterium, Navicula spp., N. cryptocephala, Nitzschia frustulum, Psammodictyon panduriforme) are mainly found at stations 1 and 5, probably as a result of their shallow depth, which allows close benthic-pelagic coupling.

Of the taxa common to all stations, some, mainly diatoms, give rise to recurrent phytoplankton blooms. The blooming species are prevalently small, often colonial, and include Chaetoceros compressus, Chaetoceros spp., Cylindrotheca closterium, Nitzschia frustulum, Skeletonema marinoi and Thalassiosira spp. Blooms

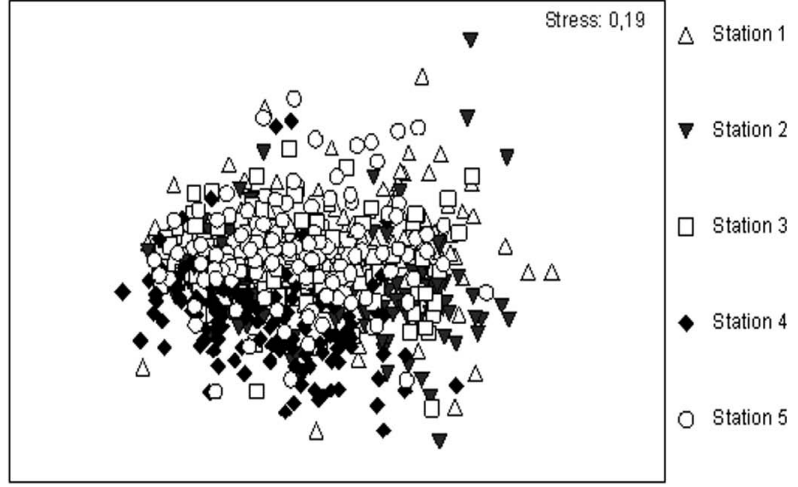

FIG. 3. - MDS plot from the matrix of 216 species and 600 phytoplankton samples; five stations are indicated by different symbols. 
TABLE 5. - A) Common species among seasonal groups; species with highest IndVal values, although not significant (n.s.), are highlighted; B) Indicator species recorded in the seasonal groups. Significant IndVal values are highlighted;

\begin{tabular}{|c|c|c|c|c|c|}
\hline & Autumn & Winter & Spring & Summer & $\mathrm{p}$ level \\
\hline \multicolumn{6}{|l|}{ A) } \\
\hline Dactyliosolen fragilissimus & 1.225 & 0.008 & 0.964 & 0.216 & n.s \\
\hline Diploneis crabro & 1.599 & 0.535 & 1.527 & 0.007 & n.s \\
\hline Psammodictyon panduriforme & 4.659 & 0.953 & 3.469 & 3.217 & n.s \\
\hline Nitzschia sigma & 2.222 & 0.555 & 0.583 & 1.576 & n.s \\
\hline Thalassionema nitzschioides & 7.659 & 2.556 & 2.842 & 4.475 & n.s \\
\hline Syracosphaera pulchra & 3.364 & 0.346 & 0.191 & 1.002 & n.s \\
\hline Asterionellopsis glacialis & 1.806 & 1.946 & 0.094 & 0.266 & n.s \\
\hline Chaetoceros decipiens & 1.171 & 1.732 & 0.443 & 0.162 & n.s \\
\hline Gomphonema olivaceum & 0.749 & 2.325 & 1.772 & 0.08 & n.s \\
\hline Gomphonema parvulum & 1.15 & 1.486 & 0.402 & 0.748 & n.s \\
\hline Fallacia forcipata & 0.347 & 2.596 & 0.121 & 0.077 & n.s \\
\hline Luticola mutica & 0.829 & 3.838 & 2.649 & 0.036 & n.s \\
\hline Pleurosigma normanii & 2.878 & 7.743 & 2.323 & 4.731 & n.s \\
\hline Pseudo-nitzschia delicatissima & 0.567 & 7.070 & 3.265 & 6.609 & n.s \\
\hline Skeletonema marinoi & 0.417 & 16.630 & 15.404 & 14.126 & n.s \\
\hline Synedra affinis & 0.678 & 2.199 & 0.126 & 0.939 & n.s \\
\hline Emiliania huxleyi & 7.905 & 13.505 & 2.513 & 3.658 & n.s \\
\hline Nitzschia conscricta & 2.594 & 2.788 & 0.778 & 2.781 & n.s \\
\hline Nitzschia longissima & 0.096 & 2.210 & 1.016 & 0.587 & n.s \\
\hline Amphora coffaeformis & 6.542 & 4.414 & 10.251 & 2.518 & n.s \\
\hline Amphora exigua & 6.025 & 7.243 & 11.136 & 6.347 & n.s \\
\hline Cerataulina pelagica & 0.044 & 0.178 & 7.066 & 3.544 & n.s \\
\hline Cocconeis placentula & 1.238 & 1.294 & 4.343 & 0.284 & n.s \\
\hline Cocconeis scutellum & 10.758 & 6.367 & 14.201 & 4.447 & n.s \\
\hline Licmophora gracilis & 0.698 & 0.911 & 2.671 & 0.098 & n.s \\
\hline Nitzchia longissima f. parva & 0.32 & 0.13 & 3.815 & 0.042 & n.s \\
\hline Rhoicosphenia abbreviata & 0.784 & 1.615 & 4.565 & 0.044 & n.s \\
\hline Nitzschia palea & 0.01 & 0.035 & 1.389 & 0.618 & n.s \\
\hline Leptocylindrus danicus & 1.047 & 1.747 & 0.236 & 2.346 & n.s \\
\hline Leptocylindrus minimus & 0.122 & 0.111 & 0.245 & 0.335 & n.s \\
\hline \multicolumn{6}{|l|}{ B) } \\
\hline Halamphora veneta & 10.891 & 1.879 & 2.925 & 1.663 & 0.01 \\
\hline Calciosolenia murrayi & 3.378 & 0 & 0 & 0 & 0.01 \\
\hline Melosira nuттuloides & 0.148 & 9.707 & 0.18 & 0.012 & 0.01 \\
\hline Pseudo-nitzschia fraudolenta & 0.084 & 7.819 & 0.123 & 0.093 & 0.01 \\
\hline Pseudosolenia calcaravis & 0 & 0.704 & 0 & 0 & 0.01 \\
\hline Navicula cryptocephala & 2.884 & 20.716 & 22.377 & 9.374 & 0.05 \\
\hline Prorocentrum minimum & 0.047 & 2.654 & 16.394 & 2.765 & 0.01 \\
\hline Eutreptia lanowii & 1.251 & 0.705 & 13.558 & 1.44 & 0.01 \\
\hline Chaetoceros tenuissimus & 0.211 & 0.017 & 6.460 & 0.81 & 0.01 \\
\hline Prorocentrum micans & 0.492 & 0.004 & 6.433 & 0.89 & 0.01 \\
\hline Entomoneis paludosa & 0.019 & 0.031 & 2.845 & 0 & 0.01 \\
\hline Cylindrotheca closterium & 0.265 & 0.891 & 7.259 & 58.209 & 0.01 \\
\hline Nitzschia frustulum & 0.351 & 0.015 & 2.923 & 41.034 & 0.01 \\
\hline Chaetoceros compressus & 0.002 & 0.302 & 0.62 & 7.123 & 0.01 \\
\hline Proboscia alata & 0.006 & 0.003 & 0.897 & 7.025 & 0.01 \\
\hline Scrippsiella trochoidea & 0.466 & 0.553 & 4.313 & 6.637 & 0.05 \\
\hline Protoperidinium bipes & 0.006 & 0.146 & 0.112 & 6.298 & 0.01 \\
\hline Scenedesmus quadricauda & 0.027 & 0.054 & 1.617 & 3.703 & 0.05 \\
\hline Guinardia flaccida & 0.012 & 0 & 0 & 1.889 & 0.05 \\
\hline
\end{tabular}

occur throughout late winter and summer $(93 \%$ from March to September). From March onwards, diatoms bloom most frequently at stations 1 and 2 . At station 2 Skeletonema marinoi was the main blooming species throughout the period. At station 1 the late winter $S$. marinoi bloom is followed by other blooms of species such as Cylindrotheca closterium, Thalassiosira spp. and Nitzschia frustulum in spring and summer. The blooms at stations 3 and 5 are much less frequent and intense. They are observed only in summer and are associated with nanoflagellates (undetermined forms) at station 3 and the tychopelagic diatom Nitzschia frustulum at station 5. At least by our definition, blooms are not recorded at station 4 .
The highest average Shannon index values (Fig. 4) are seen in ZSI 4-5. Indeed, at station 5 the community is the richest in benthic taxa $(46 \%)$, and at station 4 the phytoplankton community is characterized by the lowest frequency of a single dominant species.

\section{Seasonal phytoplankton patterns (Table 5)}

The seasonal chl $a$ pattern appears fairly uniform across all stations (Fig. 4), differing in the maximum values attained rather than in timing. The highest biomass was always recorded from May to September, with the maximum in July and a decrease in winter. The highest chl $a$ values (with a peak of $124.6 \mu \mathrm{g} \mathrm{l}^{-1}$ in 


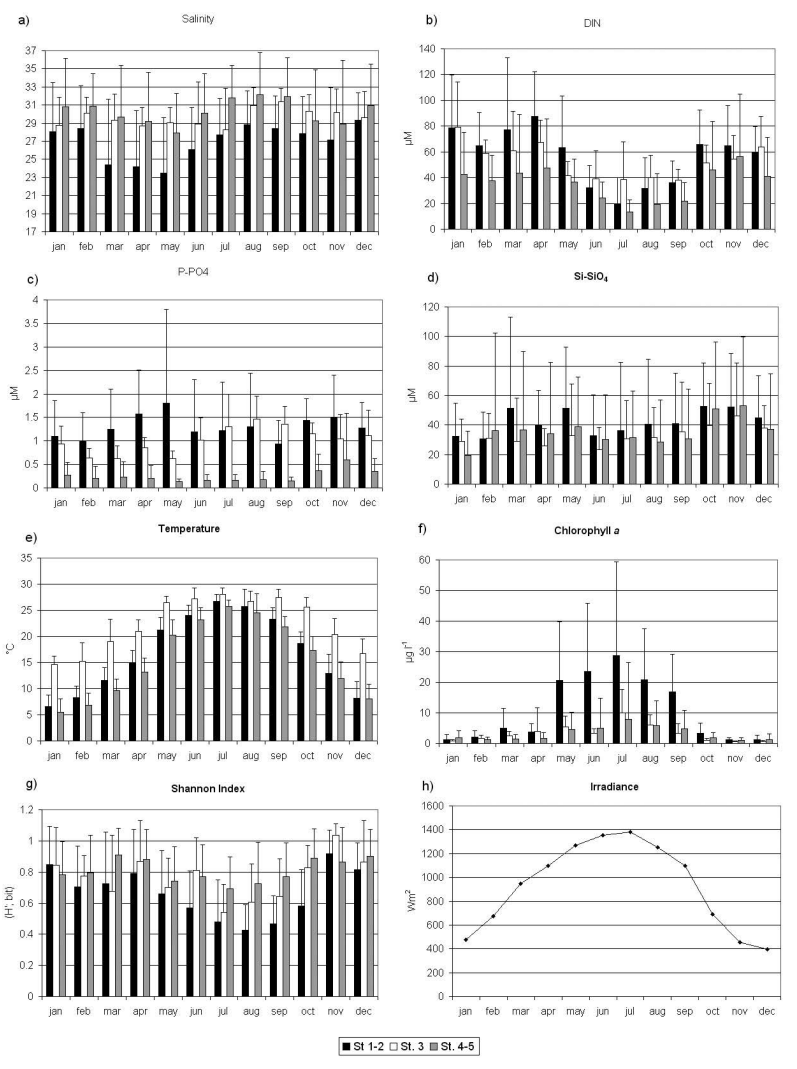

FIG. 4. - Average (bars) temporal variation and standard deviations (whiskers) at three ZSIs, of a) salinity, b) DIN, c) $\mathrm{P}_{-} \mathrm{PO}_{4}$, d) $\mathrm{Si}_{-} \mathrm{SiO}_{4}$, e) temperature, f) chl $a$, g) Shannon index; h) incident irradiance at the common measurement point

July 2001, at station 1) were attained in the ZSI with the highest nutrients (stations 1 and 2). However, at all stations the seasonal pattern of phytoplankton growth appears to be more closely related to temperature and solar irradiance than to nutrients (Fig. 4). Among nutrients we detected a clear seasonality only for DIN, with the lowest concentration in summer at every station (Fig. 4). In consideration of these general features and the common distribution of the most important taxa, described above, in this section we analyse an average seasonal cycle of the main abiotic factors, phytoplankton biomass (Fig. 4) and phytoplankton composition (IndVal analisys, Table 5).

Winter is characterized by lower temperatures, relatively low phytoplankton biomass and fairly high DIN concentrations. Phytoplankton is represented mainly by diatoms (59\% of total abundance), both benthic (Nitzschia spp. Gomphonema spp., Fallacia forcipata, Luticola mutica, Pleurosigma normanii) and planktonic (Pseudo-nitzschia spp., Skeletonema marinoi), followed by nanoflagellates (32\%); coccolithophorids attain their peak (1\%) mainly with Emiliania huxleyi. At the end of the winter, increased daylight hours and temperatures enhance phytoplankton growth. In March diatoms grow rapidly, with Skeletonema marinoi (on average $1.2 \times 10^{6}$ cells $1^{-1}$, $48 \%$ of total abundances) typically blooming from

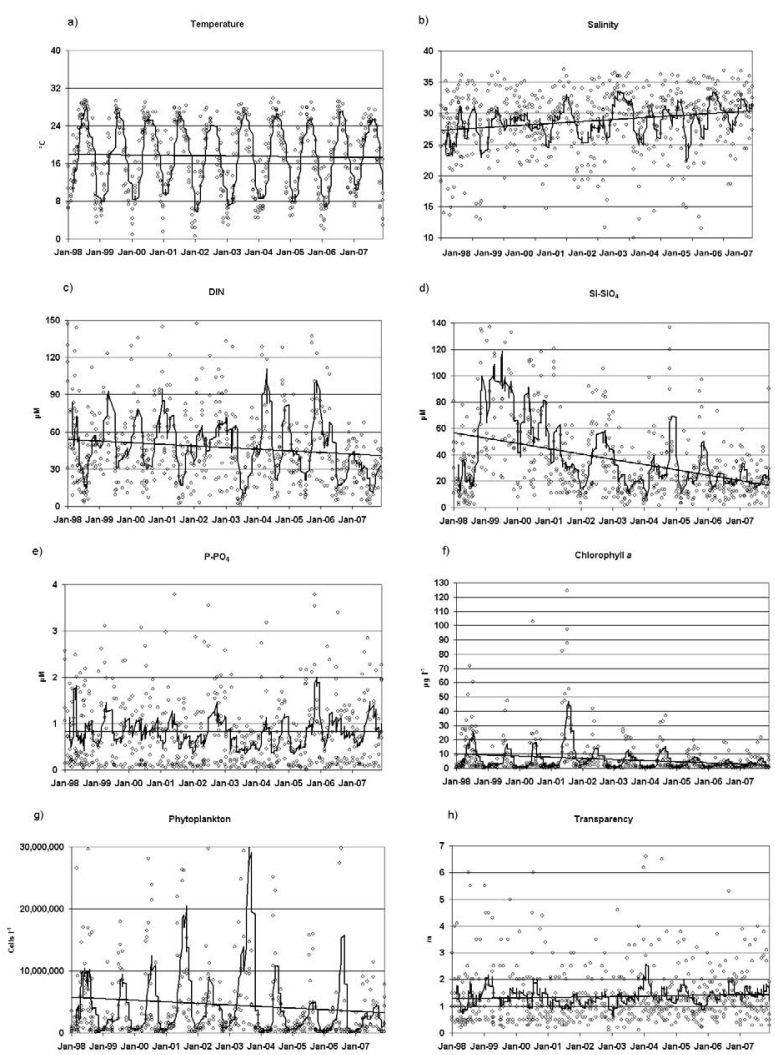

FIG. 5. - Multiannual variations of: a), temperature b) salinity, c) DIN, d) $\left.\mathrm{Si}_{-} \mathrm{SiO}_{4}, \mathrm{e}\right), \mathrm{P}_{-} \mathrm{PO}_{4} \mathrm{f}$ ) chl $a$, g) phytoplankton abundance, h) transparency. All the data are considered. In each graph the moving average and the trend are shown.

February to March not just in the Lagoon but also in the adjacent waters of the Adriatic Sea (Mozetič et al. 1998; Bastianini et al. 2004; Bernardi Aubry et al. 2006). Spring is characterized by fairly low salinity due to increased river discharge and precipitations (ARPAV 2009). A mixed community of flagellates (nanoflagellates, cryptophyceans, the dinoflagellates Prorocentrum micans and P. minimus) and euglenophyceans (e.g. Eutreptia lanowii) dominates (54\%), together with diatoms (44\%), both pelagic (e.g. Cerataulina pelagica) and benthic (e.g. Navicula spp. or Amphora spp., Cocconeis spp.). Summer sees the highest temperature and salinity and the lowest nutrient levels due to low river discharge (ARPAV 2009) and high phytoplankton uptake. In this period phytoplankton biomass peaks (average chl $a$ : $12.6 \mu \mathrm{g}^{-1}$ ). Diatoms prevail (73\%), often giving rise to blooms, mainly with Thalassiosira spp., Cylindrotheca closterium and Nitzschia frustulum. Dinoflagellates are more frequent and abundant (2\%) than in the other seasons, with Scrippsiella trochoidea, Gymnodinium spp. and Protoperidinium bipes. A drop in phytoplankton abundance and biomass accompanies the lower temperature and irradiance in autumn, despite increased nutrient availability. A mixed assemblage of flagellates (46\%) and diatoms (40\%), mainly benthic species such as Halamphora veneta, undeter- 
TABLE 6. - Seasonal Kendall test of the main abiotic parameters and phytoplankton biomass and abundance at three ZSIs: $\tau$ values, significance of trend $(p)$ and seasonal Kendall slope estimates (S.E.) in units $\mathrm{yr}^{-1}$ are shown. Statistically significant $\tau$ values are in bold.

\begin{tabular}{|c|c|c|c|c|c|c|c|c|c|}
\hline & \multicolumn{3}{|c|}{ Station 1-2 } & \multicolumn{3}{|c|}{ Station 3} & \multicolumn{3}{|c|}{ Station 4-5 } \\
\hline & $\tau$ & $p$ & S. E. & $\tau$ & $p$ & S. E. & $\tau$ & $p$ & S. E. \\
\hline Temperature $\left({ }^{\circ} \mathrm{C}\right)$ & -0.04 & 0.53 & -0.05 & 0.07 & 0.29 & 0.10 & -0.02 & 0.66 & -0.02 \\
\hline Salinity (PSU) & 0.19 & 0.04 & 0.19 & 0.32 & 0.01 & 0.31 & 0.32 & 0.01 & 0.37 \\
\hline $\mathrm{DIN}(\mu \mathrm{M})$ & -0.19 & 0.10 & -1.75 & -0.31 & 0.02 & -2.42 & -0.26 & 0.03 & -1.90 \\
\hline $\mathrm{P}_{-} \mathrm{PO}_{4}(\mu \mathrm{M})$ & -0.02 & 0.85 & -0.01 & 0.02 & 0.83 & 0.00 & -0.08 & 0.17 & 0.00 \\
\hline $\mathrm{Si}_{-} \mathrm{SiO}_{4}(\mu \mathrm{M})$ & -0.33 & 0.03 & -3.43 & -0.37 & 0.02 & -2.15 & -0.47 & 0.01 & -4.04 \\
\hline Chlorophyll $a\left(\mu \mathrm{g} \mathrm{l}^{-1}\right)$ & -0.21 & 0.06 & -0.22 & -0.26 & 0.10 & -0.07 & -0.20 & 0.15 & -0.07 \\
\hline Phytoplankton (cells $1^{-1}$ ) & -0.15 & 0.31 & -56656 & -0.25 & 0.11 & -58953 & -0.18 & 0.20 & -63054 \\
\hline
\end{tabular}

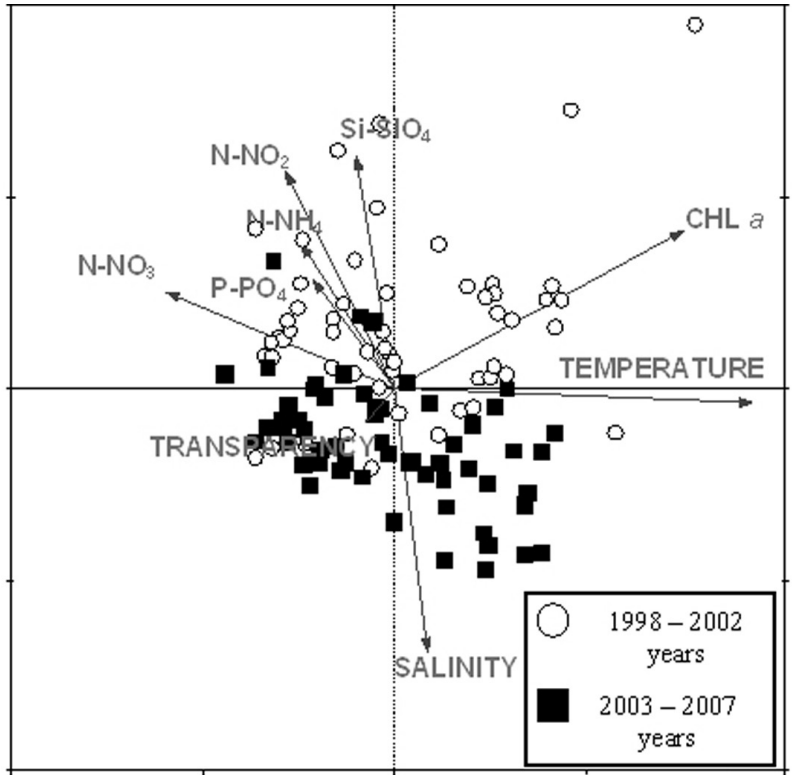

FIG. 6. - Ordination of phytoplankton data (yearly average for each station), showing differences between the periods 1998-2002 (open circles) and 2003-2007 (full squares).

mined pennate diatoms or Psammodictyon panduriforme, prevails. This period is also characterized by coccolithophorids (e.g. Calciosolenia murrayi and Syracosphaera pulcra), always with low abundance but high frequency.

The highest Shannon index (Fig. 4) is seen in autumn, as a result of a relatively high amount of species with similar low abundance, and the lowest in summer when monospecific phytoplankton blooms occur.

\section{Multiannual trends and interannual variability}

We applied the Kendall- $\tau$ test to the abiotic parameters and chl $a$ of each ZSI over the ten years. Statistically significant trends were detected only for salinity, which increased, and $\mathrm{Si}_{-} \mathrm{SiO}_{4}$, which decreased, in each ZSI (Table 6). Decreasing trends of DIN, chl $a$ and to a lesser extent phytoplankton abundance are apparent, albeit not statistically significant (Fig. 5 and Table 6).

The ordination of averaged samples (months for each year, Fig. 6) highlighted a separation between the cluster of samples belonging to the first five years (1998-2002) and that of the following five years (2003-

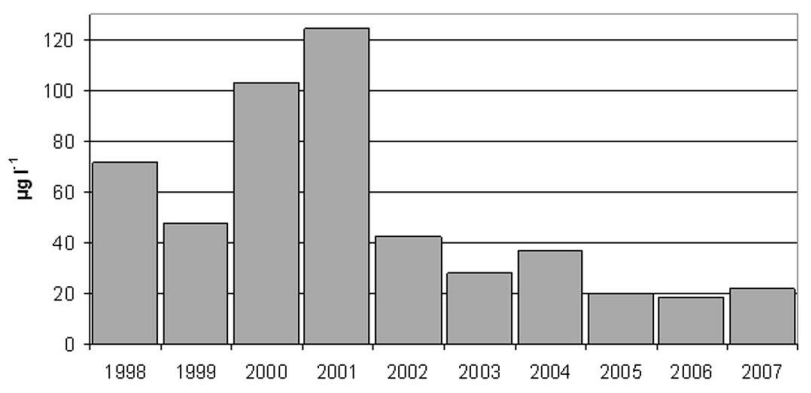

FIG. 7. - Yearly averages of chlorophyll peaks in "bloom samples".

2007), mainly related to higher nutrient concentrations, lower salinity and chl $a$ peaks in "bloom samples" in the more recent period (Fig. 7).

The medians of the two periods (Mann-Whitney test) were found to be significantly different for phytoplankton abundance, chl $a$ and the main abiotic parameters (Table 7). The significantly lower chl $a$ in

TABLE 7. - Mann-Witney test. Medians of "top ten" taxa abundance, chl $a$, diatoms, dinoflagellates, total abundance and main abiotic parameters and nutrient ratios in periods 1998-2002 (I) and 2003-2007 (II); (n.s., not significant).

\begin{tabular}{|c|c|c|c|}
\hline & I & II & p level \\
\hline Thalassiosira sp. $\left({ }^{*} 10^{6} ;\right.$ cells $\left.1^{-1}\right)$ & 51.694 & 32.438 & n.s. \\
\hline Nitzschia frustulum $\left(* 10^{6} ;\right.$ cells $\left.1^{-1}\right)$ & 15.265 & 10.659 & n.s. \\
\hline Skeletonema marinoi $\left(* 10^{6} ;\right.$ cells $\left.1^{-1}\right)$ & 32.107 & 11.533 & 0.01 \\
\hline Cyclotella sp. $\left(* 10^{6} ;\right.$ cells $\left.1^{-1}\right)$ & 16.557 & 13.983 & n.s. \\
\hline Cylindrotheca closterium $\left(* 10^{6}\right.$; cells $\left.1^{-1}\right)$ & 36.058 & 17.699 & n.s. \\
\hline Und. Cryptophyceae $\left(* 10^{6} ;\right.$ cells $\left.1^{-1}\right)$ & 56.108 & 28.967 & 0.01 \\
\hline Chaetoceros sp. $\left(* 10^{6} ;\right.$ cells $\left.1^{-1}\right)$ & 16.639 & 5.869 & 0.05 \\
\hline Navicula sp. $\left(* 10^{6} ;\right.$ cells $\left.1^{-1}\right)$ & 48.809 & 28.831 & 0.01 \\
\hline Chaetoceros compressus $\left(* 10^{6} ;\right.$ cells $\left.1^{-1}\right)$ & 4.773 & 0.501 & 0.01 \\
\hline Navicula cryptocephala $\left({ }^{*} 10^{6} ;\right.$ cells $\left.1^{-1}\right)$ & 39.302 & 15.885 & 0.01 \\
\hline Chlorophyll a $\left(\mu \mathrm{g} \mathrm{l}^{-1}\right)$ & 2.62 & 1.46 & 0.01 \\
\hline Diatoms $\left(* 10^{6} ;\right.$ cells $\left.1^{-1}\right)$ & 0.60 & 0.30 & 0.01 \\
\hline Dinoflagellates $\left(* 10^{6} ;\right.$ cells $\left.1^{-1}\right)$ & 0.01 & 0.02 & n.s. \\
\hline Total Phytoplankton $\left({ }^{*} 10^{6} ;\right.$ cells $\left.1^{-1}\right)$ & 1.64 & 0.98 & 0.01 \\
\hline Dinoflagellates/Diatoms & 0.02 & 0.04 & 0.01 \\
\hline Salinity & 28.86 & 30.66 & 0.01 \\
\hline Temperature $\left({ }^{\circ} \mathrm{C}\right)$ & 18.80 & 18.5 & n.s. \\
\hline Transparency (m) & 1.0 & 1.2 & 0.01 \\
\hline $\mathrm{N}-\mathrm{NH}_{4}(\mu \mathrm{M})$ & 12.09 & 7.8 & 0.01 \\
\hline $\mathrm{N}-\mathrm{NO}_{3}(\mu \mathrm{M})$ & 28.11 & 24.81 & 0.05 \\
\hline $\mathrm{N}-\mathrm{NO}_{2}(\mu \mathrm{M})$ & 1.55 & 1.12 & 0.01 \\
\hline $\mathrm{DIN}(u \mathrm{M})$ & 43.87 & 36.6 & 0.01 \\
\hline $\mathrm{P}_{-} \mathrm{PO}_{4}(\mu \mathrm{M})$ & 0.60 & 0.63 & n.s. \\
\hline $\mathrm{Si}-\mathrm{SiO}_{4}(\mu \mathrm{M})$ & 37.9 & 19.55 & 0.01 \\
\hline $\mathrm{N} / \mathrm{P}$ & 76.80 & 69.00 & 0.05 \\
\hline $\mathrm{Si} / \mathrm{N}$ & 0.80 & 0.50 & 0.01 \\
\hline $\mathrm{Si} / \mathrm{P}$ & 61.70 & 34.00 & 0.01 \\
\hline
\end{tabular}


the more recent period appears mainly associated with the decrease in nutrients (DIN and $\mathrm{Si}_{-}-\mathrm{SiO}_{4}$,) and the increased salinity (Fig. 5 and Table 7). The increased salinity and transparency may be linked to decreased river discharge into the Lagoon and the nearby coastal belt over the last few years (Cozzi and Giani 2011). However, despite the greater availability of light, we observed no increase in phytoplankton biomass.

While the concentrations of $\mathrm{P}_{-} \mathrm{PO}_{4}$ did not seem to fluctuate over the ten years, DIN shows a moderate decrease and $\mathrm{Si}_{-} \mathrm{SiO}_{4}$ a marked one (Fig. 5). Moreover, significant changes in the ratios between nutrients were observed. The N/P ratios, which in the Venice Lagoon and Adriatic Sea are usually much higher than the canonical 16:1 (Bianchi et al. 1999, Justić et al. 1995), were lower in the second half of the ten-year period. Because of the large fall in silicate levels, the $\mathrm{Si} / \mathrm{N}$ and $\mathrm{Si} / \mathrm{P}$ ratios diminished significantly over the decade (Table 7).

The significant fall in $\mathrm{Si}_{-} \mathrm{SiO}_{4}$ and the $\mathrm{Si} / \mathrm{N}$ and $\mathrm{Si} / \mathrm{P}$ ratios was accompanied by a decrease in the abundance of diatoms, which was significantly lower in the second half of the period, and an increase in the abundance of dinoflagellates (Table 7). However, the most abundant species (the "top ten"), including the bloom-forming ones, were always present and dominant, although with falling abundances (Table 7).

\section{DISCUSSION}

The phytoplankton of the Venice Lagoon includes taxa that are representative of the complex and open system of the Lagoon itself, from the coastal sea to the rivers and the benthic-pelagic system. Key phytoplankton species (e.g. Skeletonema marinoi, small Thalassiosira sp., Chaetoceros compressus) also bloom in the adjacent Adriatic coastal waters; others (e.g. Nitzschia frustulum, N. spp, Amphora spp) are typical lagoon inhabitants. Many of them are of benthic origin, indicating the influence of the exchange between the sediments and the water column. Finally, some species, mostly rare, are of allochthonous origin, their presence being a result of freshwater (Eutreptia lanowii, Scenedesmus quadricauda) or tidal inputs (e.g. coccolitophorids such as Syracosphaera pulchra, Rhabdosphaera claviger, Calciosolenia murrayi).

The number of phytoplankton taxa identified in this study was comparable to that recorded by Facca and Sfriso (2009) in a study with a limited time span but very large spatial coverage across the Lagoon, with diatoms and nanoflagellates as the dominant groups. The most significant species observed here were also reported by Bandelj et al. (2008) in a one-year study of six stations distributed across the whole lagoon area.

The spatial differences between the areas considered in the present study are mainly the result of the varying degrees of impact of the interactions between the adjacent marine waters, riverine inputs and benthic-pelagic coupling. Indeed, the main dissimilarities among the ZSIs (Table 3 ) were due to salinity and nutrients. The degree of benthic-pelagic coupling also determines phytoplankton composition in the different areas and seems to be mainly influenced by depth: the diatom composition (Table 4) at stations 1 and 5 (smallest depths) differs significantly in terms of the proportion of benthic species from that at stations 2 and 4 (greatest depths).

Diatoms appear to be the most representative group of the Venice Lagoon phytoplankton. They are the prevalent group in terms of abundance in most seasons and stations, and they are also representative of the riverine, coastal, benthic and pelagic influences on the system: they include species from all these systems and are also responsible for most of the blooms in the Lagoon. Indeed, diatoms dominate the nano- and microphytoplankton in other Mediterranean lagoons, such as Fusaro (Sarno et al. 1993), Varano (Caroppo 2000), Orbetello (Nuccio et al. 2003) and Stella Maris (Fanuko and Valcic 2009).

The contribution of other classes, characterizing specific periods of the year and/or sampling sites directly influenced by marine input, is rather negligible. According to Margalef's Mandala (Margalef 1978), a central paradigm of phytoplankton community ecology, the prevalence of diatoms is characteristic of permanently fertile and turbulent environments. Furthermore, the open nature of the pelagic system of many lagoons makes immigration from different systems (land, sea and sediments) an intrinsic part of their phytoplankton community structure (Cloern and Jassby 2008 and citations therein). Over the ten-year period in the Venice Lagoon, some taxa were prevalent and common (albeit with different abundances) to every station. By focusing on them, the average seasonal variation of phytoplankton composition was established, and a "phytoplankton calendar" was drawn up for the Lagoon (Fig. 8). This "calendar" may be a useful tool to evaluate alterations in the future resulting from local or global impacts that affect the timing of these key species and/or favour the dominance of new ones.

Species imported to the water column from the benthic community (benthic diatoms: Halamphora veneta, Gomphonema olivaceum, G. parvulum, Navicula cryptocephala) and from the coastal sea (coccolithophorids: Emiliania huxleyi, Syracosphaera pulcra) typify the period of lowest abundance (late autumn and winter). Planktonic diatoms mainly characterize the late winterspring start of the growing phase (Skeletonema marinoi). Moving towards the summer peaks, the species composition changes again, becoming characterized by a mixed assemblage of diatoms, which remain dominant, together with cryptophyceans, dinoflagellates and euglenophyceans. Throughout the summer period, blooms may occur, mainly of Chaetoceros compressus, small Chaetoceros spp., Cylindrotheca closterium, Nitzschia frustulum and Thalassiosira spp.

Some of these dominant species are also common to the adjacent coastal waters of the Northern Adriatic, in particular from winter to late spring: coccolithophorids 


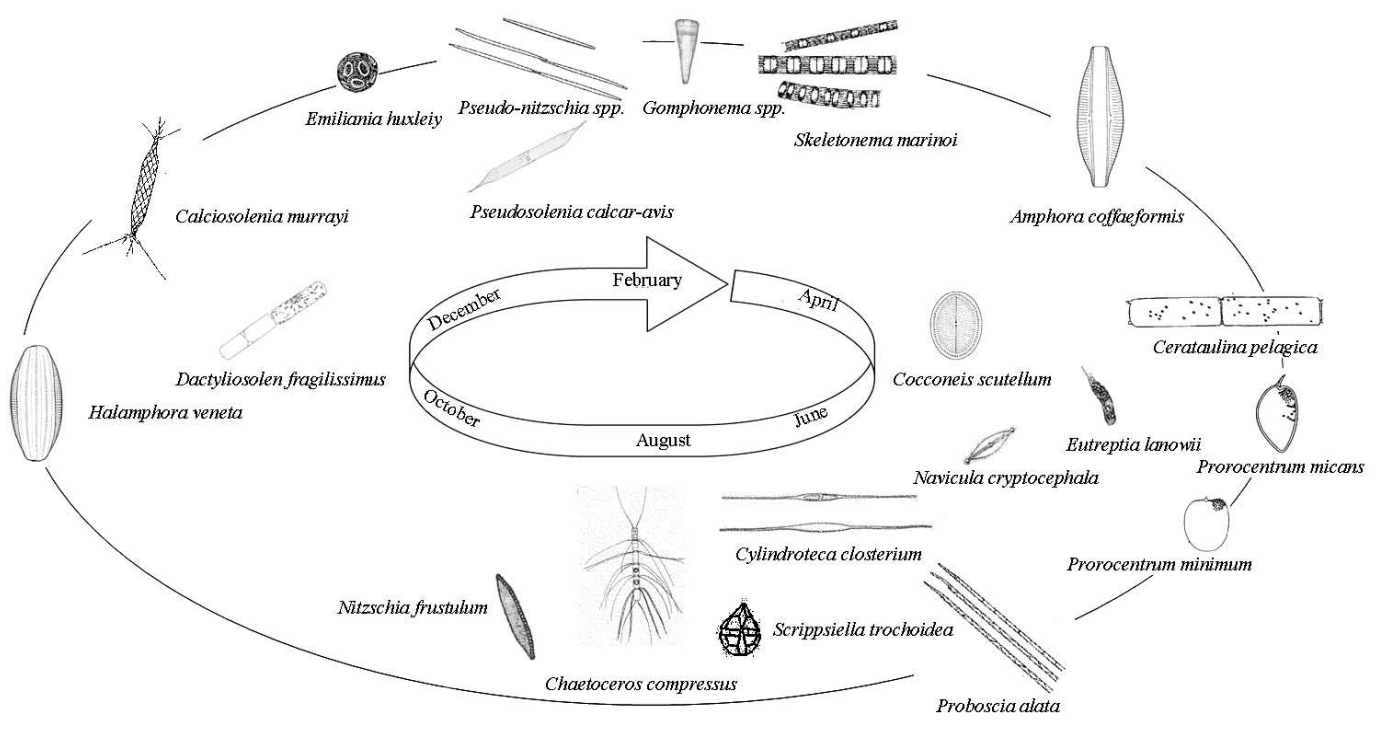

FIG. 8. - Phytoplankton calendar of Venice Lagoon; see also the text and Table 5.

and Pseudonitzscia spp in winter, Skeletonema marinoi in early spring, Cerataulina pelagica, Proboscia alata, Chaetoceros spp and small tecate dinoflagellates in late spring. Subsequently, from summer to autumn, the two communities clearly differ, with prevalence of large dinoflagellates and planktonic diatoms in the northern Adriatic (Bernardi Aubry et al. 2004).

The prevalent annual pattern of phytoplankton biomass in the Venice Lagoon is unimodal, with a summer peak, and this was seen recurrently in the ten years of study and at the five stations. This pattern appears specific to temperate enclosed coastal ecosystems, with shallow depths and permanently high nutrient concentrations (Cebrian and Valiela 1999). In contrast, the bimodal cycle of phytoplankton biomass seen in some temperate coastal ecosystems is characterized by spring blooms caused by increased light, temperature and stratification, a collapse in summer due to nutrient depletion and grazing, and autumn blooms caused by water mixing and nutrient regeneration. If nutrient availability remains high in summer, phytoplankton growth is enhanced throughout summer and is mainly linked to temperature and light.

As an ecosystem, the Venice Lagoon is constantly nutrient-enriched, apparently weakening the tight constraint on phytoplankton seasonal growth determined by nutrient limitation so that the seasonal climate cycle becomes the most recognizable driver of phytoplankton biomass. Indeed, biomass increases from spring to summer, and the maxima are generally attained in July. Within this unimodal annual cycle, which appears fairly well tuned to the seasonal cycle of temperature and irradiance, monthly fluctuations are present, highlighting the additional effect of climatic and local events occurring on shorter time scales.

A similar unimodal seasonal phytoplankton cycle is also observed in the western coastal area of the north- ern Adriatic Sea (Bernardi Aubry et al. 2004), where nutrients are lower (on average DIN from 8.0-21.6 $\mu \mathrm{M} ; \mathrm{Si}_{-} \mathrm{SiO}_{4}$ 4.8-12.0 $\mu \mathrm{M}$; $\left.\mathrm{P}_{-} \mathrm{PO}_{4} 0.10-0.24 \mu \mathrm{M}\right)$ than in lagoon waters and the chl $a$ values are on average (1.8-4.3 $\mu \mathrm{g}^{-1}$ ) close to those measured at station 4 . The phytoplankton cycle becomes much more irregular in offshore areas, with alternating minor peaks in spring and summer due to the combination of nutrient depletion and sporadic nutrient inputs (Bernardi Aubry et al. 2012): here actually both nutrients (DIN 2.8-10.9 $\mu \mathrm{M} ; \mathrm{Si}_{-} \mathrm{SiO}_{4}$ 3.1-7.3 $\mu \mathrm{M}$; $\mathrm{P}_{-} \mathrm{PO}_{4}$ 0.05-0.12 $\left.\mu \mathrm{M}\right)$ and chl $a\left(0.8-1.7 \mu \mathrm{g} \mathrm{l}^{-1}\right)$ are much lower than in the Lagoon (Bernardi Aubry et al. 2006, Socal et al. 2008).

Comparing the first half of the study period (19982002) with the second (2003-2007), a significant decrease in biomass was observed. This fall was mainly determined by a reduction in the height of the peaks and the frequency of blooms throughout the year, and was not accompanied by significant changes in species community composition or the general seasonal biomass pattern. Indeed, the same dominant species in the first five years were still among the top ten in the second five, albeit with significant reductions in abundance and biomass. Specifically, we recorded a significant decrease in diatom abundance, which could be related to the fall in silicates.

Other authors have described progressive oligotrophication over the last few years in both the Venice Lagoon (Solidoro et al. 2010) and the adjacent Adriatic Sea (Mozetič et al. 2009). In the northern Adriatic Sea a recent decrease in chlorophyll has paralleled a reduction in freshwater discharge from both the Po (Zanchettin et al. 2008) and other smaller rivers such as the Isonzo (Comici and Bussani 2007).

This trend cannot easily be interpreted in the context of other long-term studies on phytoplankton in marine coastal ecosystems. Two recent analyses of long-term 
phytoplankton trends in marine costal systems (Journal of Sea Research, 61, 2009; CIESM workshop monographs, 2010) examine time series (from one to four decades) of inshore phytoplankton and chlorophyll in relation to environmental data: these datasets do not contain significant signals that can be clearly identified and generally described and an overall conclusion is that the variability and complexity of these environments hinders the recognition of unambiguous signals.

\section{CONCLUSIONS}

Time series provide a unique tool for obtaining a reliable representation of the annual cycle of phytoplankton communities, for evaluating the existence of shifts and trends related to global or local impacts, and for testing hypotheses about phytoplankton ecology. The data series analysed in this study is only ten years long, a fairly short time span in comparison with the longest observations of phytoplankton in aquatic ecosystems, which extend over some decades, with the earliest records starting in the 1930s (Winder and Cloern 2010). Ten years are not sufficient to determine shifts and trends in annual cycles. However, they can at least provide a reliable reconstruction of annual phytoplankton variations, from both a qualitative and a quantitative viewpoint, an issue that appears particularly important for transitional areas.

Conceptualization, generalization and paradigms about the functioning of the plankton compartment are mostly available for open marine ecosystems (Margalef 1978, Longhurst 1998, Reynolds and Smayda 1998), while no wide-ranging rules have been defined for the seasonal phytoplankton cycle of coastal and transitional waters, characterized by higher overall complexity (Cloern and Jassby 2008). Our analysis of ten years of phytoplankton data in the Venice Lagoon allowed us to characterize the main features of the community. Specifically, our study revealed the following:

(i) A prevalent unimodal annual cycle of phytoplankton biomass was observed; notwithstanding some year-to-year variability and monthly fluctuations, phytoplankton biomass maxima are typically attained in summer. Annual climate fluctuations (mainly temperature and irradiance) are therefore the most recognizable driver of seasonal trends in phytoplankton biomass.

(ii) The most abundant phytoplankton taxa are common to all stations, regardless of the distinctive features of each area. It seems, therefore, that the most general ecological characteristics of the lagoon ecosystem itself (i.e. a turbulent, nutrient-enriched environment, open to other connected systems including land, sea and sediments), play a more important role in shaping phytoplankton communities than the specific attributes of each single area.

(iii) A significant decrease in biomass was observed in the second half of the period, without changes in community composition, hard to relate to a specific factor, but also seen in the adjacent Adriatic Sea. Only observations made in the next few years will allow a thorough evaluation of these changes (i.e. whether they are down to a real trend of oligotrophication or merely fluctuations).

Finally, the main results and evidence arising from the analysis of this ten-year series can be regarded as a kind of heritage for the future: a reference line against which to evaluate future changes in the phytoplankton community of the Venice Lagoon.

\section{ACKNOWLEDGEMENTS}

The authors wish to thank George Metcalf for the English revision and the two anonymous referees, who provided constructive comments for improvement to the manuscript.

\section{REFERENCES}

Acri F., Bernardi Aubry F., Bianchi F.,. Boldrin A, Comaschi A., Rabitti S. ,Socal. G. 2004. Changes in nutrients and plankton communities in the lagoon of Venice from 1970s until today. $J$. Mar. Sys. 51: 321-329.

Alberighi L., Bianchi F., Cioce F. Socal G. 1992. Osservazioni durante un bloom di Skeletonema costatum in prossimità della centrale termoelettrica ENEL di Fusina Porto-Marghera (Venezia). Oebalia 17(Suppl.): 321-322.

ARPAV, Agenzia Regionale per la Prevenzione dell'Ambiente del Veneto. 2009. Bacino scolante nella laguna di Venezia. Rapporto sullo stato ambientale dei corpi idrici. Anni 2005-2007.

Bandelj V., Socal G., Park Y., Lek S., Coppola J., Camatti E., Capuzzo E., Milani L., Solidoro C. 2008. Analysis of multitrophic plankton assemblages in the Lagoon of Venice. Mar. Ecol. Progr. Ser. 368: 23-40.

Bastianini M., Acri F., Bernardi Aubry F., Casotti R., D’Ortenzio F., Miralto A., Socal G. 2004. Environmental Factors Triggering the Late-Winter Diatom Bloom in the North Adriatic Sea. Rapp. Comm. Int. Expl. Sci. Mer Méditerranée 37: 487.

Bernardi Aubry F., Acri F. 2004. Phytoplankton seasonality and exchange at the inlets of the Lagoon of Venice (July 2001-June 2002). J. Mar. Syst. 51: 65-76.

Bernardi Aubry F., Berton A., Bastianini M., Socal G., Acri F. 2004. Phytoplankton succession in a coastal area of the NW Adriatic over a 10-years sampling period (1990-1999). Continent. Shelf Res., 24/1: 97-115.

Bernardi Aubry F., Acri F., Bastianini M., Bianchi F., Cassin D., Pugnetti A., Socal G. 2006. Seasonal and inter annual variations of phytoplankton in the gulf of Venice (Northern Adriatic Sea). Chem. Ecol. 22: 71-91.

Bernardi Aubry F., Cossarini G., Acri F., Bastianini M., Bianchi F., Camatti E., De Lazzari A., Pugnetti A., Solidoro C., Socal G. 2012. Plankton communities in the northern Adriatic Sea: Patterns and changes over the last 30 years. Estuar. Coast. Shelf Sci. 115: $125-137$

Bianchi F., Acri F., Alberighi L., Bastianini M., Boldrin A., Cavalloni B., Cioce F., Comaschi A., Rabitti S., Socal G., Turchetto M.M. 1999. Biological variability in the Venice Lagoon. In: Lasserre P., Marzollo A. (eds), The Venice Lagoon Ecosystem. Inputs and Interactions between Land and Sea. UNESCO and Parthenon Publishing Press, 97-126.

Bianchi F., Acri F., Bernardi Aubry F., Berton A., Boldrin A., Camatti E., Cassin D., Comaschi A. 2003. Can plankton communities be considered as bio-indicators of water quality in the lagoon of Venice? Mar. Poll. Bull. 46: 964-971.

Bianchi F., Socal G., Alberighi L., Cioce F. 1996. Cicli nictemerali dell'ossigeno disciolto nel bacino centrale della laguna di Venezia. Biol. Mar. Medit. 3 (1): 628-630.

Boyer J.N., Fourqurean J.W., Ronald D.J. 1997. Spatial characterization of water quality in Florida Bay by multivariate analyses: zones of similar influences. Estuaries, 4: 743-758.

Boyer J.N., Fourqurean J.W., Ronald D.J. 1999. Seasonal and long- 
term trends in the water quality of Florida Bay (1989-1997). Estuaries 22: 417-430.

Caroppo C., 2000. The contribution of picophytoplankton to community structure in a Mediterranean brackish environment. $J$. Plankton Res. 22: 381-397.

Carr M. 1996. PRIMER User Manual. Plymouth Marine Laboratory, $40 \mathrm{pp}$.

Cassie R.M. 1962. Frequency distribution model in the ecology of plankton and other organisms. J. Anim. Ecol. 31: 65-92.

Cebrian C.,Valiela I. 1999. Seasonal patterns in phytoplankton biomass in coastal ecosystems. J. Plankton Res. 21 (3): 429-444.

Clarke K.R., Warwick R.M. 1994. Change in marine communities: an approach to statistical analysis and interpretation. Plymouth Marine Laboratory, Plymouth, $144 \mathrm{pp}$.

Cloern J.E., Jassby A.D. 2008. Complex seasonal patterns of primary producers at the land-sea interface. Ecol. Lett. 11: 1-10.

Cloern J.E., Jassby A.D. 2010. Patterns and scales of phytoplankton variability in estuarine-coastal ecosystems. Estuar. Coast. 33: 230-241.

Collavini F., Bettiol C., Zaggia L., Zonta R. 2005. Pollutant loads from the drainage basin to the Venice Lagoon (Italy). Environ. Inter. 31: 939-947.

Comici C., Bussani A. 2007. Analysis of Isonzo River discharge (1998-2005) Boll. Geof. Teor. Appl. 48: 435-454.

Cozzi S., Giani, M. 2011. River water and nutrient discharges in the Northern Adriatic Sea: Current importance and long term changes. Cont. Shelf Res. 31: 1881-1893.

Cucco A., Umgiesser G. 2006. Modelling the Venice lagoon residence time. Ecol. Model. 193: 34-51.

Dufrêne M., Legendre P. 1997. Species assemblages and indicator species: the need for a flexible asymmetrical approach. Ecol. Monogr. 67: 345-366.

Edwards M., Beaugrand G., Hays G.C., Koslow J.A., Richards A.J. 2010. Trends Ecol. Evol. 25: 602-610.

Facca C., Sfriso A. 2009. Phytoplankton in a transitional ecosystem of the Northern Adriatic Sea and its putative role as an indicator for water quality assessment. Mar. Ecol., 30: 462-479

Fanuko N., Valcic M. 2009. Phytoplankton composition and biomass of the Northern Adriatic lagoon Stella Maris, Croatia. Acta Bot. Croat. 68(1): 29-44.

Gačić M., Kovačević V., Mancero Mosquera I., Mazzoldi A., Cosoli S. 2005. Water fluxes between the Venice Lagoon and the Adriatic Sea. In: Fletcher C.A., Spencer T. (eds), Flooding and environmental challenges for Venice and its lagoon: state of knowledge. Cambridge University Press, Cambridge, 431-444.

Gameiro C., Cartaxana P., Brotas V. 2007. Environmental drivers of phytoplankton distribution and composition in Tagus Estuary, Portugal. Estuar. Coast. Shelf. Sci. 75: 21-34

Guerzoni S., Rossigni P., Sarretta A., Raccanelli S., Ferrari G., Molinaroli E. 2007. POPs in the Lagoon of Venice: budgets and pathways. Chemosphere 67: 1776-1785.

Hansen H.P., Koroleff F. 1999. Determination of nutrients. In: Grasshoff K., Cremling K., Erhardt M. (eds), Methods of Seawater Analysis. Wiley-VCH Verlag: 159-228.

Heimdal B.R. 1993. Modern Coccolithophorids In: Marine Phytoplankton A Guide To Naked Flagellates And Coccolithophorids. Tanos Editors, Academic Press, pp. 147-248.

Hendey N.I. 1964. An introductory account of the smaller algae of British coastal waters. Part V: Bacillariophyceae, Diatoms. Fishery Invest. Lond. Ser. Iv 5, 317 pp.

Hirsch R.M., Alexander R.B., Smith R.A. 1991. Selection of methods for the detection and estimation of trends in water quality. Water Resour. Res. 27: 803-813.

Holm-Hansen O., Lorenzen C.J., Holmes R.W., Strickland J.D.H 1965. Fluorometric determination of chlorophyll. J. Conseil Perm. Int. Explor. Mer. 30: 3-15.

Hustedt F. 1930-1966. Die Kiesealgen Von Deutschland, Österreichs Und Der Schweiz Mit Berusichtigung Der Übrigen Länder Europas Sowie Der Angrenzender Mehresgebiete. In: Rabenhorst's Kriptogamen-Flora Von Deutschland, Österreichs Und Der Schweiz. Akad; Verlag. M.B.H. Leipzig. 7: Tl. 2. 920 Pp.: Tl., 2845 Pp.; Tl. 3, 816 pp.

Justić D., Rabalais N.N., Eugene Turner R., Dortch. Q. 1995. Changes in nutrient structure of river-dominated coastal waters: Stoichiometric nutrient balance and its consequences. Estuar. Coast. Shelf. Sci. 40: 339-356.

Legendre P. Legendre L. 1998. Numerical ecology. 2nd English edi- tion. Elsevier Science BV, Amsterdam, 853 pp.

Longhurst, A. 1998. Ecological Geography of the Sea. Academic Press, San Diego, 398 pp.

Margalef R. 1978. Life-forms of phytoplankton as survival alternatives in an unstable environment. Oceanol. Acta 1: 493-509.

Mozetič P., Fonda Umani S., Cataletto B., Malej A. 1998. Seasonal and inter-annual plankton variability in the Gulf of Trieste (northern Adriatic). J. Mar. Sci. 55: 711-722.

Mozetič P., Solidoro C., Cossarini G., Socal G., Precali R., Francé J., Bianchi F., Smodlaka N., De Vittor C., Fonda Umani S. 2009. Recent trend towards oligotrophication of the northern Adriatic: evidences from chlorophyll $a$ time series. Est. Coast. 33: 362-375

Nuccio C., Melillo C., Massi L., Innamorati M. 2003. Phytoplankyton abundance, community structure and diversity in the eutrophicated Orbetello lagoon (Tuscany) from 1995 to 2001. Oceanol. Acta 26: 15-25.

Pascher A. 1915. Clorophyceae. In: Die Susswasser Flora Deutschlands, Osterreichs und der Schweiz. Verlags von Gustav Fisher, Jena, Heft 5, 250 pp.

Peragallo H., Peragallo M. 1897-1908. Diatomees Marine de France et des Districts Maritimes Voisins. Micrographe Editeur Grez sur Loing (S. et M.), $419 \mathrm{pp}$.

Perin G. 1975. L'inquinamento chimico della Laguna di Venezia. In: Cons. depur. Acque della Z. I. Porto Marghera (ed.), Problemi dell'inquinamento lagunare. Venezia: 47-89. Rampi L., Bernhardt M. 1980. Chiave Per La Determinazione Tassonomica Delle Peridinee Pelagiche Mediterranee: C.N.E.N., Roma (Rt/B10(80) 8): 1-193.

Rampi L., Bernhardt M. 1981. Chiave Per La Determinazione Tassonomica Delle Coccolitoforidee Pelagiche Mediterranee: C.N.E.N., Roma (Rt/B10(81) 13): 1-98.

Reynolds C.S., Smayda T.J. 1998. Principles of species selection and community assembly in the phytoplankton: Further exploration of the mandala: pp. 8-10. In: Reguera B., Blasco J., Fernandez M.L., Wyatt T. (eds), Harmful Algae. Xunta de Galicia and Intergovernmental Oceanographic Commission of UNESCO, Paris.

Sarno D., Zingone, A., Saggiomo V., Carrada G.C. 1993. Phytoplankton biomass and species composition in a Mediterranean coastal lagoon. Hydrobiologia, 271: 27-40.

Schiller J. 1931-37. Dinoflagellatae (Peridineae) Monografischer Behandlung. In: Rabenhorst Kriptogamen-Flora Von Deutschland, Österreichs Und Der Schweiz. Verlag. M. B. H. Leipzig. 10(3) -1, 1-617, (1931-1933), (10)3-2, 1-590, (1933-1937).

Sen P.K. 1968. Estimates of the regression coefficient based on Kendall's tau. JASA, 63: 1379-1389.

Sfriso A., Facca C. 2007. Distribution and production of macrophytes in the lagoon of Venice. Comparison of actual and past abundance. Hydrobiologia, 577: 71-85

Sfriso A., Facca C., Ceoldo S., Marcomini A. 2005. Recording the occurrence of trophic level changes in the lagoon of Venice over the '90s. Environ. Int., 31: 993-1001.

Shannon C.E., Weaver G. 1949. The mathematical theory of communication, vol. III. Univ. Illinois Press, Urbana, IL, $125 \mathrm{pp}$.

Sin Y., Wetzel R.L., Anderson I.C. 1999. Spatial and temporal characteristics of nutrient and phytoplankton dynamics in the York River estuary, Virginia: Analysis of long-term data. Estuaries, 22: $260-275$.

Socal G., Ghetti, L., Boldrin, A., Bianchi, F. 1985. Ciclo annuale e diversità del fitoplancton nel porto-canale di Malamocco. Laguna di Venezia. Atti - Ist. Veneto Sci. Lett. Arti 143: 15-30

Socal G., Bianchi, F., Comaschi, A., Cioce, F. 1987. Spatial distribution of plankton communities along a salinity gradient in the Venice lagoon. Arch. Oceanogr. Limnol. 21: 19-43.

Socal G., Bianchi F., Alberighi L. 1999. Effects of thermal pollution and nutrient discharges on a spring phytoplankton bloom in the industrial area of the lagoon of Venice. Vie Milieu 49: 19-31.

Socal G., Acri F., Bernardi Aubry F., Berton A., Bianchi F., Capuzzo E., Coppola J., Facca C. Sfriso A. 2006. Analisi dei popolamenti fitoplanctonici nella laguna di Venezia dal 1977 al 2004. Biol. Mar. Medit. 13: 178-184.

Socal G., Acri F., Bastianini M., Bernardi Aubry F., Bianchi F., Cassin D., Coppola J., De Lazzari A., Bandelj V., Cossarini G., Solidoro C. 2008. Hydrography and biogeochemical features in the northern Adriatic Sea during the period 2003-2006. Mar. 
Ecol. 29: 449-468.

Sokal R., Rohlf J. 1981. Biometry, second ed Freeman, San Francisco, $859 \mathrm{pp}$

Solidoro C., Bandelj V., Bernardi Aubry F., Camatti E., Ciavatta S., Cossarini G., Facca C., Franzoi P., Libralato S., Melaku Canu D., Pastres R., Pranovi F., Raichevic S., Socal G., Sfriso A., Sigovini M., Tagliapietra D., Torricelli P. 2010. Response of Venice Lagoon ecosystem to natural and anthropogenic pressures over the last 50 years. In: Kennish M.J., Paerl H.W. (eds), Coastal lagoons: critical habitats of environmental change. CRC Press, Boca Raton, FL, 483-511.

Solidoro C., Melaku Canu D., Cucco A., Umgiesser G. 2004. A partition of the Venice lagoon based on physical properties and analysis of general circulation. J. Mar. Sys. 51: 147-160

Sournia A. 1986. Atlas du phytoplancton marin. Editions du Centre National de la Recerche Scientifique, 1: 1-219, 2: 1-297.

Strickland J.D.H., Parsons T.R. 1972. A practical handbook of seawater analysis. Bull. Fish. Res. Board Can., 167: 1-310.

Tagliapietra D., Zanon V., Frangipane G., Umgiesser G., Sigovini M. 2009. Physiographic zoning of the Venetian Lagoon. In: Campostrini P. (ed.), Scientific Research and Safeguarding of Venice, vol. VII - 2007-2010 results: 161-164.

Theil H. 1950. A rank-invariant method of linear and polynomial regression analysis, 1, 2 and 3. Nederlandse Akademie Wetenschappen Proceedings, 53: 386-392, 521-525, 1397-1412.

Tolomio C., Moschin E., Moro I., Andreoli C. 1999. Phytoplankton de la Lagune de Venise. I: bassins nord et sud (avril 1988 - mars 1989). Vie Milieu 49: 33-44.

Tolomio C., Bullo L. 2001. Prelievi giornalieri di fitoplancton in una stazione del bacino meridionale della laguna di Venezia; aprile 1993 - marzo 1994. Boll. Mus. Civ. Stor. Nat. Venezia 52: 3-23.

Tomas C.R., Hasle G.R. 1997. Identifying marine phytoplankton. Academic Press, New York, 858 pp.

Throndsen J. 1993. The planktonic marine flagellates. In: Marine phytoplankton a guide to naked flagellates and coccolithophorids. Tanos Editors, Academic Press, pp. 7-131.

Uthermöhl H. 1958. Zur Vervollkomnung der quantitativen Phytoplankton-Methodik. Mitt. Int. Ver. Limnol. 9: 1-38.

Voltolina D. 1975. The phytoplankton of the lagoon of Venice: November 1971 - November 1972. Pubbl. Staz. Zool. Napoli 39: $206-340$.

Winder M., Cloern J.E. 2010. The annual cycles of phytoplankton biomass. Phil. Trans. R. Soc. B. 365: 3215-3226

Zanchettin D., Traverso P., Tomasino M. 2008. Po river discharge: a preliminary analysis of a 200 year time series. Clim. Chang. 89: 411-433.

Zingone A., Totti C., Sarno D., Cabrini M. Caroppo C., Giacobbe M.G., Luglié A., Nuccio C., Socal G. 2010. Fitoplancton: metodiche di analisi quali-quantitativa. In: Socal G., Buttino I., Cabrini M., Mangoni O., Penna A., Totti C. (eds), Metodologie di studio del plancton marino. Manuali e Linee Guida 56/2010 ISPRA, SIBM Roma: pp 213-237.

Zuliani A., Zaggia L., Zonta R. 2005. Freshwater discharge from the drainage basin to the Venice lagoon (Italy), Environ. Int. 31: $929-938$.

Scient. ed.: M. Estrada.

Received April 16, 2012. Accepted September 27, 2012.

Pubñished online January 21, 2013. 